\title{
Metaphysical explanations and the counterfactual theory of explanation
}

\author{
Stefan Roski ${ }^{1}$
}

Published online: 20 August 2020

(C) The Author(s) 2020

\begin{abstract}
According to an increasingly popular view among philosophers of science, both causal and non-causal explanations can be accounted for by a single theory: the counterfactual theory of explanation. A kind of non-causal explanation that has gained much attention recently but that this theory seems unable to account for are grounding explanations. Reutlinger (Eur J Philos Sci 7(2):239-256, 2017) has argued that, despite these appearances to the contrary, such explanations are covered by his version of the counterfactual theory. His idea is supported by recent work on grounding by Schaffer and Wilson who claim there to be a tight connection between grounding and counterfactual dependence. The present paper evaluates the prospects of the idea. We show that there is only a weak sense in which grounding explanations convey information about counterfactual dependencies, and that this fact cannot plausibly be taken to reveal a distinctive feature that grounding explanations share with other kinds of explanations.
\end{abstract}

Keywords Grounding · Metaphysical explanation · Counterfactuals

\section{Introduction}

For a long while, the notion of explanation figured in metaphysical debates mainly in connection with questions of theory choice, explanatory power being one dimension along which competing metaphysical theories can be compared. Since a couple of years, however, metaphysicians have become interested in explanation from a different, more direct perspective. In particular, the view that there are

Stefan Roski

stefan.roski@gmail.com

1 Philosophisches Seminar, Universität Hamburg, Überseering 35, Postfach \#4, 22297 Hamburg, Germany 
certain distinctively metaphysical explanations has become increasingly popular. Arguably the most prominent cases in point (though not the only ones) are explanations based on grounding. ${ }^{1}$ Pertinent examples include explanations of logically complex truths in terms of their simpler constituents (e.g.: Socrates is Greek and wise partially because he is wise), explanations of truths about determinables in terms of truths about corresponding determinates (e.g.: The taxi is colored because it is yellow), and explanations of truths about mental properties in terms of truths about neurological properties (e.g.: Kim is in pain because Kim's c-fibres are firing). While it is widely acknowledged that there are such explanations, there is no consensus on how they relate to explanations that have mostly been at issue in the pertinent debates in the philosophy of science, i.e. explanations based on causal relations (e.g.: The species became extinct due to the prevalence of predators). In a recent paper, Reutlinger (2017) has argued that both types of explanations can be accounted for by the same theory of explanationindeed, by a theory that follows the highly popular counterfactual approach to explanation. Reutlinger's proposal is part of a growing trend in the philosophy of science, where a number of authors have recently argued that Woodward's (2003) counterfactual account of causal explanation can be modified to also account for various kinds of non-causal explanations. ${ }^{2}$ The apparent success of these attempts motivates a view according to which not only causal, but all explanations, work by providing information about patterns of counterfactual dependence between entities mentioned in explanans and explanandum. What would make this view particularly attractive is that it promises an informative monist account of explanation which pinpoints a non-trivial feature that is common to a wide variety of otherwise different explanations, including grounding explanations. ${ }^{3}$

As attractive as the view appears to be, however, it is not viable as it stands. As I will argue in this paper, grounding explanations do not work by conveying information about patterns of counterfactual dependence. The reason for this is simple: a large number of paradigmatic cases of grounding either do not give rise to counterfactual dependence, or do so only in a rather weak sense which does not plausibly highlight any theoretically interesting property of explanations.

The structure of the paper is as follows. In Sect. 2, I will go over the motivation to endorse counterfactual approaches to explanation in general and for grounding explanations in particular. Subsequently, I will present a precise statement of Reutlinger's proposal for a monist counterfactual theory of explanation and show

\footnotetext{
1 See, among others, Audi (2012), Rosen (2010), Schaffer (2016) and Schnieder (2011). Two remarks: (1) I write 'based on grounding' as I favour a view according to which grounding is not identical to a kind of explanation, but rather supports or backs explanations; I thus favour separatism about grounding in the sense of Raven (2015). Most of what I say, however, could be adapted to a position where 'x grounds y' would express a sense of ' $x$ explains y'. (2) Not every distinctively metaphysical explanation need be grounding-based. Glazier (2017), for instance, argues that there are metaphysical explanations based on essences that differ from grounding explanations.

2 See, among others, Baron et al. (2017), Baron (2019), Bokulich (2011), Rice (2015), Reutlinger (2016), Povich (2018), Povich (2019) and Saatsi and Pexton (2013).

3 For arguments in favour of a monist theory of explanation, see Schaffer (2016, 89f), Schnieder (2011) and Nickel (2010).
} 
that it successfully accounts for some paradigmatic grounding explanations. In Sects. 3 and 4, I will discuss problems. While the problems discussed in Sect. 3 pertain to the specific version of the account developed by Reutlinger, the problems discussed in Sect. 4 pertain to the general idea of counterfactual approaches to explanation that aim to account for grounding-based explanations. In Sect. 5, I will conclude with some brief observations about the consequences of my conclusions for monism about explanation.

\section{Counterfactual approaches to explanation and Reutlinger's theory}

\subsection{Preliminaries}

Before we begin, let me make a number of assumptions explicit that will be in the background of what follows (mostly for the sake of definiteness). First, I will assume that grounding is an asymmetric, transitive relation between facts, where facts are construed as relatively fine grained and abundant. In particular, I assume there to be disjunctive, existential, and negative facts, and I moreover assume there to be different necessarily obtaining facts. Second, I take the relata of the explanation-relation (i.e. the relation between explanans and explanandum) to be true propositions or collections thereof, where propositions are taken to be relatively fine-grained. In particular, I assume there to be different necessarily true propositions. Third, I assume that whether or not the explanation relation obtains is an entirely objective matter, in the same sense in which it is, for instance, an objective matter whether or not Goldbach's Conjecture is true. While this is a controversial view, it is assumed by my opponents in this paper and I will grant it to them for the sake of the argument. Finally, a remark on the central ingredient of all counterfactual approaches to explanation: the notion of counterfactual dependence. It is important to keep in mind that the term 'counterfactual dependence' is not used unequivocally by all adherents of counterfactual approaches to explanation. Depending on how it is understood, different counterfactual approaches to explanation will make different predictions in some cases. ${ }^{4}$ While Woodward favours an interventionist account of counterfactuals (2003, Ch. 3), other authors work with (variations of) possible-worlds approach(es), and yet others take the general idea of the theory to be compatible with multiple different approaches to counterfactual dependence. Reutlinger, whose theory we will focus on below, rejects interventionism as a general account of counterfactual dependence, since he (with Woodward 2003, 221) considers it ill-suited to account for non-causal forms of explanatory dependence..$^{5}$ At the same time, Reutlinger takes the motivation and the general idea of counterfactual approaches to explanation to be largely separable from particular views about counterfactuals and compatible with various (non-

\footnotetext{
${ }^{4}$ See Briggs (2012) for an informative comparison of an interventionist and a similarity-based semantics that points out differences with regards to truth-conditions and evaluability.

5 See, e.g. Reutlinger (2016, 738). For Reutlinger's own account of counterfactuals that he takes to be a direct competitor to Woodward's, see Reutlinger (2013, Ch. 8).
} 
interventionist) accounts (2016, 736, 2017, 244, 2018, 78). Throughout the paper, I will follow Reutlinger in this assumption and not presuppose any particular account of counterfactuals. Where specific assumptions about counterfactuals become relevant, I will signal it.

\subsection{The main idea of the theory}

The existence of explanations based on grounding is widely acknowledged. However, it is not fully clear how such explanations relate to more familiar types of explanations such as, for instance, causal explanations. Reutlinger (2017) has argued that they can be covered by an account that takes as its starting point a theory which has dominated the debate on explanation in the last couple of decades, namely Woodward's (2003) counterfactual theory of causal explanation. In this section, I will go over the main idea and motivation for this latter theory, and then present Reutlinger's take on it.

According to Woodward's theory, explanations work at least partially because they make patterns of counterfactual dependence explicit. In making such patterns explicit, explanations provide answers to a certain type of question, so-called whatif-things-had-been-different questions. As Woodward puts it:

an explanation ought to be such that it can be used to answer what I call a what-if-things-had-been-different question: the explanation must enable us to see what sort of difference it would have made for the explanandum if the factors cited in the explanans had been different in various possible ways (Woodward 2003, 6).

Given the widely accepted view that events counterfactually depend on their causes, it is straightforward to see how this idea works in the case of causal explanations. In the simplest case of an explanation of a given token event $E$, citing a cause $C$ of $E$ answers the question what would have been different had $C$ not occurred: $E$ would not have occurred either. ${ }^{6}$

Importantly, however, and as stressed by Woodward, many causal explanations that occur in scientific contexts do not merely provide the relatively unspecific information that a certain event mentioned in the explanandum would not have occurred had some event mentioned in the explanans not occurred. (Cf. Woodward 2003 , 215ff.) Oftentimes, such explanations also provide information about how precisely things would have been different with the event mentioned in the explanandum, had things been different with the event(s) mentioned in the explanans in some specific way or other. For instance, if a given event $E$ involves some magnitude having a certain value, and an explanation of $E$ 's occurrence cites some initial conditions and a law, then one can typically use the law to show how precisely the value of the magnitude associated with $E$ would have changed, had the

\footnotetext{
6 As cases of preemption and symmetric overdetermination show, this will not be true for all choices of $C$ and $E$. Again, different theories of counterfactuals will evaluate such problematic cases differently, but I take it to be uncontroversial that any account of counterfactual dependence should deliver the same predictions for simple causal dependencies.
} 
initial conditions been different. A bit more concretely, consider an explanation of why a car drives at $80 \mathrm{mph}$ that cites the driver's pushing the gas pedal with a specific force $f$, some information about the internal workings of the car, and certain laws of mechanics. In such a case, the respective laws will provide information about how the car's speed would have been different had the driver pushed with a specific, different force $f^{\prime}$. In that sense, the explanation does not merely provide information about what would have happened had the event mentioned in the explanans not occurred, but also about what exactly would have happened had a range of specific, alternative events occurred. As we will see below, counterfactuals that relate specific alternatives to the explanans to specific alternatives to the explanandum are important to account for grounding explanations in terms of a counterfactual approach.

Thus far I have illustrated the general idea of counterfactual approaches to explanation for the case of causal explanations. This is the case for which Woodward originally developed his theory. ${ }^{7}$ However, a number of authors have recently argued that the basic framework can be extended so as to also be applicable to various non-causal explanations. ${ }^{8}$ Why should it specifically apply to grounding explanations? Some support for this idea comes from recent work on grounding. Both Schaffer (2016) and Wilson (2018a, b) have proposed accounts of grounding according to which there is a tight connection between grounding and counterfactual dependence. (Not incidentally, both accounts are largely inspired by Woodward's counterfactual theory of causation.) Wilson has tried to make the case that grounding should be understood as metaphysical, as opposed to nomological, causation. Part of his case is the view that "both grounding and nomological causation are closely associated with distinctive patterns of one-way counterfactual dependence" (Wilson 2018b, 25). And while Schaffer does not claim that grounding is causation, he argues that grounding and causation share important similarities, one of which is that both give rise to counterfactual dependence. He suggests, for instance, a "working test of token grounding [...] in terms of counterfactual covariation: wiggle the ground, and the grounded wiggles" (Schaffer 2016, 74).

It is somewhat unclear whether Schaffer and Wilson want to claim that every case of grounding gives rise to counterfactual dependence, or merely a large class of paradigmatic cases. In any case, we shall see that by 'counterfactual dependence' they presumably mean something weaker than what is ordinarily understood by this

\footnotetext{
7 Note that Woodward acknowledges other types of explanations besides causal ones. However, he does not claim that they can all be treated in his version of the counterfactual theory. In that sense, he is a pluralist about explanation: contrary to Reutlinger and other monists, he does not think that all explanations exhibit patterns of counterfactual dependence. As mentioned above, one reason for this is that he favours an interventionist account of counterfactuals and does not think that this account is applicable to non-causal forms of explanatory dependence. Woodward (2003, 221), he even suggested that not being interpretable in terms of interventionist counterfactuals is a characteristic feature of noncausal explanations.

${ }^{8}$ See the references in footnote 2 .
} 
term. ${ }^{9}$ But be that as it may, even if there are cases where grounding does not give rise to counterfactual dependence (in any sense of the term), this is not necessarily a problem for counterfactual approaches to explanation. For, such approaches aim to provide accounts of explanation and not of grounding. And it might simply be the case that not all cases of grounding underwrite explanations but merely those in which the grounded fact counterfactually depends on the respective grounds. (Indeed, that every case of causation gives rise to counterfactual dependence is a highly contested claim, the truth of which the counterfactual approaches are not generally taken to be hostage to.) Counterfactual dependence would thus figure as a criterion of explanatory relevance, filtering out explanatorily relevant grounds from those that are irrelevant (cf. Tan 2019, 39f). Hence, merely pointing to a specific case of grounding that does not give rise to counterfactual dependence is generally insufficient to falsify the counterfactual theory.

So, at least if Schaffer and Wilson are right about grounding, it might seem initially plausible to assume that Reutlinger's counterfactual theory of explanation is applicable also to grounding explanations. The following optimistic statement by Reutlinger thus might appear warranted:

[I]t is only mildly speculative to assert that the [counterfactual theory of explanation] generalizes to all grounding explanations, if the explanatory grounding facts exist as a matter of metaphysical contingency (Reutlinger 2017, 250).

Before we move on to discuss Reutlinger's theory in detail, let me briefly comment on the restriction he makes in the above passage. Reutlinger restricts the scope of his theory to cases where the grounds mentioned in a given explanans do not obtain necessarily. He does this presumably to avoid trivialization. For, at least according to the standard account of counterfactual dependence, any fact vacuously depends counterfactually on any arbitrary necessarily obtaining fact (and this will also be true for any of the weakened versions of counterfactual dependence we shall consider below). On the standard account, $g$ counterfactually depends on $f$ just in case $g$ wouldn't obtain were $f$ not to obtain. But on the Lewis/Stalnaker semantics for counterfactuals, this latter claim will be vacuously true whenever $f$ obtains necessarily. ${ }^{10}$ So, at least on the standard account of counterfactual dependence,

\footnotetext{
9 Schaffer proposes his working test of token grounding by extrapolating from a similar test for token causation (Schaffer 2016, 74). With respect to causation, he admits that the test is only applicable "in a restricted simple range of cases" $(2016,64)$. He also points to cases of grounding, where the test does not work, but claims that "plausible extensions" will work $(2016,79)$. Wilson also mentions some cases where grounding does not give rise to counterfactual dependence as it is ordinarily understood, but suggests that there are weaker kinds of counterfactual dependence that always come along with grounding (Wilson 2018b, §5). I will come back to these cases below in Sect. 4. On the question to what extent one may assume that there is a unified notion of counterfactual dependence which adherents of the counterfactual theory may rely on, see also footnote 19 below.

${ }^{10}$ While this problem does not straightforwardly arise in an interventionist framework, there are serious questions regarding the idea of intervening on necessarily obtaining circumstances. The assumption that there are such interventions is indeed often deemed incoherent; see Weslake (forthcoming, section 3.3) and cf. Woodward (2003, 112). In any case, as mentioned above, Reutlinger rejects interventionist accounts of causation; Reutlinger $(2016,738)$.
} 
counterfactual approaches do not seem to be able to distinguish between explanations and non-explanations whenever the explanans mentions a necessarily obtaining fact. However, one may argue that this is not so much a problem for these approaches, but rather for the standard account of counterfactual dependence (cf. Tan 2019). Indeed, most adherents of counterfactual approaches to non-causal explanation assume there to be non-vacuous relations of counterfactual dependence between necessarily obtaining facts. ${ }^{11}$ And even though there is no stable theory of such relations at this point, it seems fair to follow Reutlinger and set explanations in terms of necessarily obtaining facts aside, which I will do for the remainder of this paper.

However, even if one concentrates on cases where "the explanatory grounding facts exist as a matter of metaphysical contingency" (ibid.), there remains a related problem. Many plausible candidates for grounding explanations are explanations of necessarily obtaining facts in terms of contingent ones. For instance, it is widely assumed that grass's being green or not green is grounded in, and explained by, grass's being green. But it seems that counterfactual approaches cannot tolerate such explanations. For, no necessarily obtaining fact can depend counterfactually on any contingently obtaining fact. ${ }^{12}$ As far as I know, neither Reutlinger nor any other adherent of a counterfactual approach to non-causal explanation discusses this problem. That being said, there is a potential response to the problem that adherents of counterfactual approaches might find congenial. One may argue that contingent grounds of necessary facts are not explanatorily relevant. While necessarily obtaining facts are grounded in contingently obtaining facts, the reply would go, they are explained only by other necessarily obtaining facts such as logical or metaphysical laws. The idea in the background here would be that contingent facts do not have enough explanatory oomph to fully account for necessarily obtaining ones. ${ }^{13}$ While the contingent fact that grass is green fully grounds the necessary fact that grass is green or not green, to explain why grass is green or not green one has to refer to some necessarily obtaining fact, e.g., the law that, for every fact, either it or its negation obtains. In that case, of course, accounting for such explanations within a counterfactual theory would again require some theory of non-trivial counterfactual dependence among necessary facts. So, more work would have to be done to spell out this idea in detail. For the sake of this paper, though, I will grant also this point to adherents of the counterfactual approaches to non-causal explanation and from now on concentrate on cases of grounding where all facts involved obtain contingently.

\footnotetext{
11 See, e.g., Baron et al. (2017) and Povich (2019).

12 For, if $g$ obtains necessarily, then it obtains in every world and so there is no fact $f$ such that if $f$ weren't to obtain, $g$ wouldn't obtain. Something similar also holds for any the weaker versions of counterfactual dependence that we'll consider below.

13 Intuitions of this kind seem to play a role in the discussions around Blackburn's Dilemma; cf. Cameron (2010).
} 


\subsection{Statement of Reutlinger's account}

Thus far we have discussed the general idea of counterfactual approaches to explanation, but no precise statement of any particular account. Indeed, most adherents of the idea leave their accounts at a relatively informal level. Reutlinger is a welcome exception to this. ${ }^{14}$ In a number of papers, he has made his account precise as follows. We let propositions $G_{1}, \ldots, G_{m}$ be generalizations, $S_{1}, \ldots, S_{n}$ propositions that state initial and auxiliary conditions, and $E$ the explanandum. According to Reutlinger's counterfactual theory of explanation, $G_{1}, \ldots, G_{m}, S_{1}, \ldots, S_{n}$ explain $E$ if and only if the following three conditions are satisfied: ${ }^{15}$

1. Veridicality condition: $G_{1}, \ldots, G_{m}, S_{1}, \ldots, S_{n}$, and $E$ are (approximately) true.

2. Implication condition: $G_{1}, \ldots, G_{m}$ and $S_{1}, \ldots, S_{n}$ logically entail $E$ or a [nonzero, SR] conditional probability $P\left(E \mid S_{1}, \ldots, S_{n}\right)$-where the conditional probability need not be 'high' in contrast to Hempel's covering law account.

3. Dependency condition: $G_{1}, \ldots, G_{m}$ support at least one counterfactual of the form: had $S_{1}, \ldots, S_{n}$ been different than they actually are (in at least one way deemed possible in the light of the generalizations), then $E$ or the conditional probability of $E$ would have been different as well (Reutlinger 2017, 244). ${ }^{16}$

Three comments on this: First, for what follows, neither the Veridicality Condition, nor the provisions about conditional probability will play any role. Second, Reutlinger does not impose any requirements on the generalizations $G_{1}, \ldots, G_{m}$ apart from them being non-accidentally true (Reutlinger 2017, 244, fn 9). Third, although Reutlinger claims that his account "largely follows" $(2017,244)$ the one by Woodward (2003, 203), there is a crucial difference between both (over and above the fact that Woodward's account is restricted to causal explanations). While Woodward's account is formulated in the framework of structural equation models and against the background of an interventionist construal of counterfactuals, Reutlinger wants to commit to neither of these. And indeed, whether non-causal explanations are susceptible to being modelled by this machinery is controversial. ${ }^{17}$

\footnotetext{
14 As noted by Khalifa et al. $(2018, \S 2)$.

15 I am slightly altering Reutlinger's original formulation. He takes the explanation relation to obtain between sentences, while I take it to obtain between propositions. I do this mainly to simplify presentation.

16 Cf. also Reutlinger (2016, (2018).

17 For skepticism concerning the notion of intervention in cases of non-causal explanations, see Khalifa et al. (2018) and Saatsi and Pexton (2013). For problems with structural equation models in non-causal contexts, see Jansson (2018) and Koslicki (2016).
} 
So, in line with Reutlinger, I will neither assume an interventionist construal of counterfactuals nor the structural equations framework. ${ }^{18}$ For most of what follows, we can rely on our philosophically informed, but otherwise pre-theoretical understanding of counterfactuals (though I will occasionally put claims about counterfactual dependence in terms of possible worlds, since it is heuristically useful). ${ }^{19}$

With this out of the way, let us move on to see how the theory accounts for a number of paradigmatic examples for grounding explanations.

\subsection{Success cases}

As is commonly assumed, given that $\{$ Mars $\}$ exists, the fact that $\{$ Mars $\}$ exists is fully grounded by the fact that Mars exists. It is also commonly assumed that such cases of grounding underwrite grounding explanations: \{Mars\} exists because Mars exists. This seems perfectly in line with Reutlinger's theory. We can safely assume the Veridicality Condition to be satisfied. The Implication Condition should be satisfied as well. That Mars's existence comes along with $\{$ Mars\}'s existence is no accident. The existence of any given object necessitates the existence of its singleton. So, there is a non-accidental generalization which, in conjunction with the truth that Mars exists, logically entails the truth that $\{$ Mars $\}$ exists. Finally, also the Dependency Condition is satisfied: if Mars wouldn't exist, \{Mars $\}$ wouldn't exist

\footnotetext{
18 Two referees have raised the question whether Reutlinger's non-interventionist counterfactual theory of explanation is still in the spirit of Woodward's original account. After all, since both authors endorse different views on the semantics of counterfactuals, their theories might lead to different predictions even in the realm of causal explanations. Since my criticism below will be mainly concerned with Reutlinger's theory and not with Woodward's, this question is tangential to the main argument of the present paper. That being said, I'd like to stress that Woodward and Reutlinger endorse two core ideas that underlie each of their accounts of explanation, namely the idea that (a) explanations make explicit how specific ways for the explanans to be different relate to specific ways for the explanandum to be different (they answer what-if-things-had-been-different-questions), and that (b) such relations between ways to be different can be expressed in terms of counterfactuals. Indeed, Reutlinger himself takes the core idea of Woodward's account to be independent of the latter's specific theory of counterfactuals. See Reutlinger (2016, 735-736, 2018, 78). Notably, Woodward has defended (a) and (b) in earlier works independently of an interventionist account of counterfactuals; cf. Woodward (1979, 54-55). Also in later works he takes (a) to be an idea that holds both for causal and non-causal explanations (2003, 221), despite the fact that he takes his interventionist theory of counterfactuals only to be properly applicable to causal explanations.

19 I should stress that this is a non-trivial assumption-though one that is in favour of my opponents in this paper. For it is unclear whether there is a robust pre-theoretical notion of counterfactual dependence that is applicable to causal and non-causal cases alike. In addition to that and as mentioned earlier, different semantic paradigms for counterfactuals evaluate them in different ways, leading to different assignments in truth-value, different inferential behaviour, and even to differences with respect to the question whether certain counterfactuals are evaluable in the first place. (See, again, Briggs (2012) for a recent discussion of differences between interventionist and similaritybased accounts.) And even if we grant Reutlinger that his theory is compatible with a variety of different counterfactual dependence accounts of causation (Reutlinger 2017, 251), it is unclear whether any of these accounts can be extended to also cover non-causal forms of counterfactual dependence.
} 
either. Plausibly enough, this counterfactual is supported by the generalization involved in our explanation. ${ }^{20}$

There are further success cases. The fact that snow is white and the fact that grass is green jointly ground the conjunctive fact that snow is white and grass is green. And there is a corresponding grounding explanation of the conjunctive fact in terms of its conjuncts. Also this case is covered by Reutlinger's account. We can, again, safely assume the Veridicality Condition to be satisfied. The Implication Condition is satisfied as well. Conjunctive facts are necessitated by their conjuncts, taken jointly. And so there is a non-accidental generalization that, together with the truth that snow is white and the truth that grass is green, logically entails the truth that snow is white and grass is green. Finally, the Dependency Condition is satisfied: if snow were not white or grass were not green, it would not be the case that snow is white and grass is green. ${ }^{21}$ Plausibly enough, this counterfactual is supported by the generalization involved in our explanation.

\section{Problem-cases I: the Implication Condition}

The success cases show that Reutlinger's version of the counterfactual theory yields the right results at least sometimes. However, brief reflection on the cases also shows that the account has some implausible consequences on the assumption that the conditions it specifies are jointly sufficient for the explanation relation to obtain. Two problems can be brought out by reflecting on the Implication Condition. ${ }^{22}$ These problems might not reveal fundamental flaws of the counterfactual theory of explanation in general, but they point to some crucial problems of Reutlinger's specific articulation of it. And since it is at least not obvious how to reformulate the account in such a way that it avoids the problems, it is worth taking a closer look at them.

A circularity worry. Earlier (Sect. 2.4), I have claimed that there is a nonaccidental generalization which, in conjunction with the truth that Mars exists, logically entails the truth that $\{$ Mars $\}$ exists - as is required by the Implication Condition. A plausible way to put this generalization is as follows:

\footnotetext{
${ }^{20}$ Since Reutlinger does not specify what exactly he means by 'counterfactual support', this last point is not as straightforward as it may seem. Reutlinger merely mentions a necessary condition for a generalization to support a particular counterfactual, namely that the generalization be non-accidentally true (Reutlinger 2017, 244, fn 9). This condition is satisfied in the case at hand. Moreover, since the counterfactual in the present case is a necessary truth, it is vacuously entailed by whatever generalization appears the explanans. But one might conceivably construe the relation of counterfactual support to be something more demanding than mere entailment. Here and in what follows, however, I will grant Reutlinger that the requirement of counterfactual support is satisfied.

21 The example that Reutlinger discusses in his paper $(2017, \S 4)$ is fundamentally of the same sort. He discusses explanations of why a given truth is a Humean law in terms of the collection of instances of the law (the Humean mosaic), and the conditions for being a law according to the Best System Account. As he presents the case, this explanation relies (at least in part) on the fact that universal generalizations are grounded in their instances. This idea, in turn, is usually viewed as a generalization of the idea that conjunctive facts are grounded in their conjuncts; see, e.g., Fine $(2012,59)$.

22 Further problems for the sufficiency of the account are pointed out by Khalifa et al. (2018).
} 
(Singleton-Generalization) For any $x$, if $x$ exists, $\{x\}$ exists.

The problem is that non-accidental generalizations like this come very cheap. Next to (Singleton-Generalization), there is also:

(Reverse-Singleton-Generalization) For any $x$, if $\{x\}$ exists, $x$ exists.

Together with the truth that \{Mars $\}$ exists, this claim logically entails the truth that Mars exists. And since Mars's existence counterfactually depends on \{Mars\}'s existence, there seems to be an explanation of the former in terms of the latter according to Reutlinger's theory. Now, this will surely not be a grounding explanation since grounding is asymmetric. Still, it satisfies all conditions of Reutlinger's account and thus counts as an explanation. But this means that Reutlinger's conditions allow for explanatory circles. The truth that Mars exists helps explain a truth that helps explain the truth that Mars exists (and similarly for any other explanation of this singleton-member type). I take this to be a highly undesirable result. Even if one is generally open to the existence of some explanatory circles (as Reutlinger 2017, 253), such circles should not be that prevalent and that easy to establish. ${ }^{23}$

It is conceivable that this problem can be circumvented by imposing additional conditions on the generalizations admissible in explanations or by working with a more demanding conception of counterfactual dependence. ${ }^{24}$ To develop such a conception, however, is a burden adherents of the counterfactual theory have to carry.

An overgeneration worry. Even if we bracket the aforementioned circularityproblem, Reutlinger's conditions are implausibly permissive. To see this, suppose that some initial conditions $I$ and a generalization $G$ pass Reutlinger's conditions for some given explanandum $E$. If this is the case, then $I, G$, and the non-accidentally true generalization that everything is self-identical will also pass the conditions. Since logical consequence is non-monotonic, adding the truth that everything is self-identical to a given explanans will not lead to a failure of the Implication Condition. Nor will it lead to a failure of the Dependency Condition. For, it will surely not prevent the generalization in the explanans from supporting the counterfactual pertinent to the explanation in question. Hence, $I, G$, and the truth that everything is self-identical will count as an explanation for $E$. But clearly, the truth that everything is self-identical is not in general explanatorily relevant to every truth that has an explanation at all.

In order to prevent cases like this, one could slightly modify the Implication Condition such that each part of the explanans ought to be necessary for the explanandum to be logically deducible from it. ${ }^{25}$ However, if this is how the account is to be understood, a problem emerges. This problem can be brought out by

\footnotetext{
${ }^{23}$ Note that Reutlinger seems to assume that metaphysical explanations are generally asymmetric (Reutlinger 2017, 253).

24 Indeed, A. Wilson takes it that an interventionist construal of counterfactual dependence can circumvent this type of problem; see Wilson $(2018 \mathrm{a}, \S \S 4,5)$. His corresponding remarks remain, however, programmatic.

25 Cf. Reutlinger (2018, 79).
} 
reflecting on the second example mentioned above: the explanation of a conjunction in terms of its conjuncts. In this case, no generalization is necessary to ensure the truth that grass is green and snow is white to be logically deducible from the truth that grass is green and the truth that snow is white. But this means that if we understand Reutlinger's conditions in such a way that they exclude deductively redundant generalizations from being part of the explanans, the explanation at hand does not satisfy the conditions after all. And similar remarks apply to any explanation that is based on what is often called logical grounding, i.e. grounding explanations of logically complex truths in terms of their simpler constituents. For, in any such case, the respective groundee is logically entailed by its ground. ${ }^{26}$

So, it seems that Reutlinger's version of the counterfactual theory of explanation either countenances far too many explanations to be acceptable (due to the Implication Condition being too liberal), or it entails that no case of logical grounding underwrites an explanation (due to a failure of a suitably restricted version of the Implication Condition). Again, it seems conceivable that suitable reformulations of the account could prevent this problem, but it is not obvious how such reformulations might look like, and the burden of spelling out the details lies with adherents of the counterfactual theory.

\section{Problem cases II: the Dependency Condition}

While I do think the problems mentioned in the previous section cast serious doubt on the viability of Reutlinger's specific formulation of the counterfactual theory of explanation, I do not think this is a fatal blow for his project, or for the counterfactual theory in general. After all, there have been very few successful attempts to characterize philosophically central phenomena in terms of explicitly stated necessary and sufficient conditions. Why should explanation be different in this respect? It would be interesting enough if the counterfactual theory, in Reutlinger's version, merely specified conditions that are necessary for the explanation-relation to obtain and which point to a non-trivial feature that grounding explanations share with other types of explanations. There are, however, several other cases in which the crucial condition of the counterfactual theory-the Dependency Condition-does not seem to be satisfied. I will consider two kinds of cases. The first kind are grounding explanations of the instantiation of properties that can be realized in multiple, mutually incompatible ways. The paradigm case of this are explanations of why some object has a determinable property that cite a corresponding more determinate property. Structurally related cases are (at least on some accounts) explanations of mental facts in terms of physical facts and explanations of dispositions in terms of their categorical bases. A second kind of case are explanations of facts that are overdetermined by their grounds. Paradigmatic examples are explanations of disjunctive facts in terms of their disjuncts, where more than one disjunct obtains, or explanations of why there are certain $F \mathrm{~s}$

${ }^{26}$ See e.g. Correia and Schnieder $(2012,21)$. 
that cite one among many particular $F$ s. In neither of these cases can we expect the fact cited in the explanandum to counterfactually depend on the facts cited in the explanans in any ordinary sense of 'counterfactual dependence'. In Sects. 4.1 and 4.2, we shall see that there is a way to understand Reutlinger's Dependency Condition on which it is nonetheless satisfied in those cases. In Sect. 4.3, however, I shall argue that understanding the condition this way comes at the cost of trivializing it.

\subsection{Determination}

Facts involving determinable properties are generally assumed to be grounded in facts that involve corresponding, more determinate properties. For instance:

(1) The fact that rose $r$ is scarlet grounds the fact that $r$ is red.

Does (1) underwrite a grounding explanation according to the counterfactual theory? The Implication Condition is clearly satisfied. Facts involving determinates of a given determinable property necessitate facts involving the latter. So, there is a non-accidental generalization that, in conjunction with the truth that $r$ is scarlet (and truths to the effect that being scarlet is a determinable of being red), logically entails the truth that $r$ is red. What about the Dependency Condition? At first glance, it might seem that a case like (1) poses serious trouble for the condition. For, it is doubtful that the following counterfactual is true:

(2) Had $r$ not been scarlet, $r$ would not have been red.

Indeed, on the Lewis/Stalnaker semantics, (2) seems false. The closest worlds in which $r$ is not scarlet will surely encompass worlds in which it has some other shade of red.

Note, however, that (2)'s not being true does not show that Reutlinger's Dependency Condition is not satisfied. For, the condition does not require the truth of a counterfactual of the form 'had $S_{1}, \ldots, S_{n}$ not been the case, $E$ wouldn't have been the case'. It merely requires that the generalizations of the explanation "support at least one counterfactual of the form: had $S_{1}, \ldots, S_{n}$ been different than they actually are (in at least one way deemed possible in the light of the generalizations), then $E[\ldots]$ would have been different as well" Reutlinger (2017, 244). ${ }^{27}$ In other words, the counterfactual theory merely requires that some counterfactual of a certain type be true: a counterfactual whose antecedent mentions some specific way for the initial conditions to be different. And there are such counterfactuals in the case at hand, for instance:

(3) If rose $r$ were beige, it would not be red.

(4) If rose $r$ were navy, it would not be red.

27 Cf. also Reutlinger $(2018,79)$. I do not assume that Reutlinger requires the counterfactuals in question to have precisely the form he indicates in the above quote, i.e. such that the antecedent existentially quantifies over ways of being different. In any case, the counterfactuals I'll consider below entail counterfactuals of precisely the form indicated above. 
(5) If rose $r$ were navy, it would be blue.

So, at least on an intuitive level, there seem to be ways for the initial conditions of our grounding explanation to be different on which the explanandum is different as well. Further, it seems plausible that counterfactuals (3)-(5) are supported by the generalizations involved in the explanans. ${ }^{28}$ In that case, the Dependency Condition is fulfilled, and the explanation satisfies all of Reutlinger's conditions. The same should hold for nearly all cases of grounding that involve determinate-determinable links. ${ }^{29}$ And if the conditions are fulfilled in cases of grounding that involve determinate-determinable links, they should also hold for analogous cases mentioned earlier, i.e. (on some views) explanations of dispositions in terms of their categorial bases and explanations of facts about mental states in terms of facts about physical states.

So, it seems that cases of grounding that involve determinable properties (or other multiply realizable properties) do not pose a problem for Reutlinger's conditions. Let us next see how the conditions cope with other cases of grounding that do not give rise to ordinary counterfactual dependence.

\subsection{Overdetermination}

Many facts are overdetermined by their grounds. Typical cases in point are existentially quantified facts that have multiple obtaining instances. Before we consider whether the CTE can deal with such cases, we need to get a clearer grip on the kinds of counterfactuals that are pertinent to the Dependency Condition. In the case of determinates and determinables, the condition was arguably satisfied. The counterfactuals witnessing this in our example case [(3)-(5)] are in a certain sense more specific than counterfactuals witnessing ordinary counterfactual dependence. Their evaluation requires us to consider relatively specific ways for the initial conditions of a given explanation not to obtain rather than them simply not obtaining. However, while it was relatively clear in the case above what such specific ways for conditions not to obtain amount to, it is not clear in general.

In Woodward's counterfactual theory of causal explanation, ways for initial conditions of an explanation not to obtain correspond to results of idealized interventions that change values of variables in a structural equation framework. ${ }^{30}$ However, as mentioned earlier, Reutlinger does not want to make any commitment to this part of Woodward's account (and for good reasons, see footnote 17 above). But what else shall we, in general, understand by a way for conditions to be different? For present purposes, I propose to take ways to be different to correspond

\footnotetext{
28 Again, if one were to take entailment to be sufficient for counterfactual support, (3)-(5) would clearly be supported by the generalizations in our explanans, since (3)-(5) are all necessarily true.

29 Possible exceptions concern determinables that occur in necessarily obtaining facts, which I exclude from consideration; cf. Sect. 2.2 above.

30 See Woodward (2003, Ch. 2).
} 
to possible worlds. To consider some condition $C$ to be different in some specific way $C^{\prime}$ means to consider a world where $C^{\prime}$ does, and $C$ does not, obtain. ${ }^{31}$

With this in the background, let us consider how the counterfactual theory copes with explanations of overdetermined facts. Consider the fact that there are musicians. According to virtually every account of grounding, this fact is grounded, for instance, in the fact that Clapton is a musician:

(6) That Clapton is a musician grounds that there are musicians.

It is, again, widely assumed that such grounding facts give rise to grounding explanations. ${ }^{32}$ Clearly, however, the following counterfactual is false:

(7) If Clapton weren't a musician, there would be no musicians.

In other words, the fact that there are musicians does not counterfactually depend on Clapton's being one in the ordinary sense of the term. The former fact is overdetermined by its grounds, and radically so. Still, there is some way for our explanans to be different that will lead to the explanandum being different. For, given our previous proposal to understand ways for conditions to be different, any world in which there are no musicians corresponds to a way for the condition of Clapton's being a musician to be different on which the fact that there are musicians does not obtain. And while the worlds closest to ours are worlds in which there are musicians, there are also worlds, and hence ways for the initial conditions of the explanation in question to be different, in which there are none. ${ }^{33}$ In particular, if we let $C$ be a condition that is satisfied precisely in the worlds in which there are no musicians, the following counterfactual comes out true: ${ }^{34}$

\footnotetext{
31 As a general characterization of ways to be different, the proposal is presumably too coarse grained in some respects, and too fine grained in others. It is too fine grained in that it distinguishes between different ways for, say, the condition of Socrates being wise to be different that differ merely in minor details having nothing to do with Socrates or his wisdom (e.g. the precise temperature on Alpha Centauri tomorrow noon). It might be too narrow in that it cannot distinguish between different ways for conditions not to obtain whenever those conditions necessarily do not obtain. These points do not matter for the cases at hand, however.

32 Incidentally, that existential quantifications are explained by their instances is a thesis that Lewis (1986, 223) defended, quite independently from any considerations about grounding. Of course, adherents of the view that existentially quantified facts (such as the fact that there are musicians) are explained by their instances are by no means committed to the view that there aren't also other explanations of such facts (e.g. an anthropological or socio-cultural explanation of why there are musicians).

${ }^{33}$ On an interventionist interpretations of counterfactuals, such cases are not easily dealt with. There does not seem to be a coherent conception of intervention on which one can intervene on Clapton's being a musician in such a way that there are no musicians.

34 This way to cope with ground-theoretic overdetermination is to a certain extent similar to the way to cope with symmetric, causal overdetermination in structural equation frameworks by relativizing counterfactual dependence to values of variables in a "redundancy range"; cf. Hitchcock (2001, 289290) and Woodward (2003, 83-84). Abstracting from details of the structural-equation framework, the basic idea of this approach is as follows. An event $E$ that is causally overdetermined by the events $C_{1}, \ldots, C_{n}$ can be said to counterfactually depend on each $C_{i}$ (with $1 \leq i \leq n$ ) in the sense that under the condition that the causal ties between $E$ and each of the other events $C_{j}$ (with $1 \leq j \leq n$ and $i \neq j$ ) are severed and $C_{i}$ wouldn't occur, $E$ wouldn't occur. Conditions like the one expressed by the antecedent of
} 
(8) If Clapton weren't a musician and $C$ obtained, there would be no musicians.

Are such counterfactuals supported by the generalizations employed in the relevant explanations, as required by Reutlinger's Implication Condition? Plausibly, the generalizations that are involved in grounding explanations of existential quantifications will track the truth-conditions of the latter (cf. Fine 2010, 105f). And such generalizations do seem to support counterfactuals like (8).

It deserves to be mentioned at this point that even though the explanation we have considered is somewhat of a toy-example, it instantiates a structure that is found also in more interesting cases. Plausibly, that someone is a criminal is grounded in, and can be explained by, them committing some given crime. Suppose Robin has committed many crimes: he robbed a bank, committed tax-fraud, etc. Each of these crimes, individually, suffices to metaphysically explain why Robin is a criminal. On the present understanding of ways to be different, the counterfactual theory confirms this. While Robin would still be a criminal if he hadn't committed any particular of his crimes taken individually, there is a true counterfactual of the following form:

(9) If Robin hadn't robbed the bank and $D$ obtained, Robin wouldn't be a criminal.

where $D$ is a condition that holds in precisely those worlds in which Robin did not commit any crimes.

\subsection{Trivialization}

We have seen that the conditions of Reutlinger's counterfactual theory of explanation are satisfied in a wide range of grounding explanations, even in cases where the respective explananda do not counterfactually depend on the explanantia in the ordinary sense of the term. The theory achieves this by focussing on more specific counterfactuals: counterfactuals that encode specific ways for conditions mentioned in the explanans to be different which come along with specific ways for conditions mentioned in the explanandum to be different. And while Reutlinger does not give any explication of what such ways to be different are supposed to be, we have found one straightforward construal of this notion that is wide enough to account for many standard examples of grounding explanations. However, while this saves Reutlinger's account from obvious counterexamples, it also puts the idea that the account identifies a unifying feature of different kinds of explanations in jeopardy.

First, that grounding explanations track counterfactual dependencies in the sense that they entail claims such as (8) or (9) is not a particularly informative feature. After all: for any two contingent facts $f$ and $g$, there is a counterfactual of the form 'if $f$ would not obtain and $C$ obtained, then $g$ would not obtain'. Simply pick any

Footnote 34 continued

such a counterfactual correspond to ways for $C_{i}$ 's occurrence to be different in the sense specified above they are specific ways for $C_{i}$ not to occur. 
condition incompatible with $g$ for $C$. Since $g$ is contingent, we are guaranteed that there will be such a condition. But a notion of counterfactual dependence that obtains between any two contingent facts can surely not be said to pick out a feature that informatively distinguishes explanatory relations from any other kind of relation between contingent facts. At best, it seems, a counterfactual such as

(10) If neither Clapton nor anyone else were a musician, there would be no musicians.

is a cumbersome way to express that a given existentially quantified fact would not obtain if none of its instances obtained. But this kind of information is already carried by the generalizations (the metaphysical laws) which are involved in a grounding explanation of why there are musicians. A counterfactual like (10) does not seem to add any explanatorily relevant information to these generalizations. Consequently, it is at the very least unclear whether the Dependency Condition identifies a non-trivial feature which grounding explanations share with, e.g., causal explanations. Even though this does not strictly speaking render the theory false, it considerably weakens Reutlinger's case for explanatory monism.

Second, and related to the first point, if grounding explanations merely give rise to a kind of counterfactual dependence that is encoded by claims such as (8) and (9), it becomes unclear how the counterfactual account can distinguish between explanatorily relevant and explanatorily irrelevant information. At the beginning of the paper, I have mentioned that in the realm of causal explanations counterfactual dependence is often offered as a criterion that distinguishes explanatorily relevant causal influences from those that are irrelevant. ${ }^{35}$ As some have argued, such distinctions can also be made with respect to grounding explanations; that is, one can sensibly distinguish between grounds that are explanatorily relevant to a given grounded fact and those that aren't. ${ }^{36}$ It is a desideratum for a theory of grounding explanations to account for this distinction. However, to the extent that the counterfactual theory has to invoke the weak notion of counterfactual dependence that we have discussed above, it is unable to do this.

An obvious rejoinder to the problems mentioned above would be to argue that the construal of Reutlinger's Dependency Condition that I have proposed is simply too weak and should be replaced by a stronger construal. Taking a cue from Lewis's late theory of causation as influence (Lewis 2000, 190), a natural suggestion would be not to allow the Dependency Condition to be satisfied on any way for the initial conditions to be different, but only on 'not too distant' alterations to them. Accordingly, adherents of the counterfactual theory could replace the Dependency Condition by the following one:

Narrow Dependency Condition: The generalizations involved in our explanation support at least one counterfactual of the form: had the initial conditions of the explanans $S_{1}, \ldots, S_{n}$ been different than they actually are (in at least one way

\footnotetext{
35 See Tan (2019, 39) and Hitchcock (1995, 311).

36 See Krämer and Roski $(2017, \S 5)$.
} 
deemed possible in the light of the generalizations) and within a range of not too distant alternations to $S_{1}, \ldots, S_{n}$, then $E$ would have been different as well.

This way, the theory would still capture the idea that explanations answer what-ifthings-had-been-different-questions by providing counterfactual information about conditions for the explanandum to be different on which the explanans is different as well. However, the kind of counterfactual dependence required by the Narrow Dependency Condition is non-trivial: it clearly does not obtain between any two contingent facts.

Unfortunately, though, on this understanding of the counterfactual theory, it can no longer account for many grounding explanations. Depending on how exactly one wants to spell out 'not too distant', the condition may well be satisfied in the determinate-determinable case. Plausibly, considering a world where an object is navy blue rather than light red is a not too distant alteration of the initial conditions. However, in the case of overdetermined facts such as the fact of there being musicians, or of someone being a criminal, etc. the conditions are not generally satisfied: a world with no musicians is surely too distant an alternative if anything is. But these latter cases are paradigmatic examples for grounding explanations.

It is conceivable that the Narrow Dependency Condition can be weakened in such a way that it accounts for all grounding explanations while not being trivially satisfied. However, it is far from straightforward to see how this could be done. In any case, the burden of proof lies with adherents of counterfactual approaches to explanation.

\section{Conclusion: consequences for explanatory monism}

Reutlinger proposes his counterfactual theory as a monist theory of explanation, a theory that identifies features that are shared by causal, grounding, and other explanations. He argues that one such feature is a certain kind of counterfactual dependence that holds between entities mentioned in explanans and explanandum. However, on the most straightforward reading of his Dependency Condition, the kind of counterfactual dependence that his theory requires to hold holds between any two contingent facts. And on straightforward ways of strengthening the condition, it is violated by paradigmatic grounding explanations. Either way, the condition is unhelpful in identifying a feature that grounding explanations share with other kinds of explanations. Note also that, while I have focussed on Reutlinger's specific account, the problems I have mentioned pose challenges for any account that tries to cash out explanatory power in terms of counterfactual dependence. Recall, moreover, that we have already made substantial concessions to adherents of the counterfactual theory. Even bracketing the aforementioned problems, a full-blown monist version of the counterfactual theory should be able to account for explanations that involve necessarily obtaining facts, and thus requires an account of non-trivially true counterpossibles, which is thus far still a desideratum. 
The prospects of a monist counterfactual theory of explanation do thus not look bright. This does not mean, however, that the project of developing a monist account of explanation has to be jettisoned altogether. Let me conclude with some brief remarks on what an alternative version of explanatory monism might look like.

Abstracting a bit from Reutlinger's formulations, we can view his theory as a version of the backing model of explanation that has been influentially proposed by Kim (1988, 1994) and Ruben (2012). According to this model, all explanations work because they provide information about objective relations of determination or dependence such as causation or grounding. The backing model of explanation is commonly considered a monist theory of explanation: all explanations instantiate a common template, even though they can differ with respect to the kind of relation that backs them. Reutlinger's version of the counterfactual theory can be viewed as an attempt to give some more substance to this idea by identifying features that explanation-backing relations have in common, namely (a) giving rise to a certain form of counterfactual dependence, and (b) being governed by non-accidental generalizations. ${ }^{37}$ We have seen that (a) is no plausible candidate for a distinctive feature of explanation-backing relations. (It might be a good candidate for a feature that distinguishes causation from other explanation-backing relations.) Feature (b), on the other hand, looks more promising. After all, relations that are governed by non-accidental generalizations do not obtain between any two contingent facts. Being connected by such relations thus seems a promising candidate for a unifying feature of explanation-backing relations. Indeed, it has been argued recently that grounding is law-governed in precisely the same sense in which causation is. ${ }^{38}$ That being said, being governed by non-accidental generalizations is by itself not sufficient for being an explanation-backing relation. Relations such as identity, necessitation, or supervenience are governed by non-accidental generalizations but are generally considered unable to back explanations. Explanation-backing relations pose more demanding constraints. Plausible further requirements are being directed in some way (i.e. being non-symmetric) and having a certain modal force. ${ }^{39}$ Causation and grounding both have these traits. So, a promising idea would be to develop a monist account of explanation based on the idea that explanations provide information about, modally robust, law-governed relations of directed dependence. To develop such an account, however, is work for another occasion.

Acknowledgements For comments on previous versions of this article, I am grateful to Stephan Krämer, Martin Glazier, and the audience at Benjamin Schnieder's Forschungskolloquium: Sprache und Welt in November 2018. I'd also like to thank three anonymous referees for this journal for their helpful comments. Work on this paper was funded by the Deutsche Forschungsgemeinschaft through an Emmy Noether grant for the project Relevance (Grant KR 4516/2-1). I am very grateful for this support.

Funding Open Access funding provided by Projekt DEAL.

\footnotetext{
37 Schaffer sometimes comes close to endorsing a similar position, see Schaffer (2016, 89-90, 2017, §§2.1-2.2).

38 See e.g., Schaffer (2017) and Rosen (2017).

39 Cf. Bennett (2017, Ch. 2).
} 
Open Access This article is licensed under a Creative Commons Attribution 4.0 International License, which permits use, sharing, adaptation, distribution and reproduction in any medium or format, as long as you give appropriate credit to the original author(s) and the source, provide a link to the Creative Commons licence, and indicate if changes were made. The images or other third party material in this article are included in the article's Creative Commons licence, unless indicated otherwise in a credit line to the material. If material is not included in the article's Creative Commons licence and your intended use is not permitted by statutory regulation or exceeds the permitted use, you will need to obtain permission directly from the copyright holder. To view a copy of this licence, visit http:// creativecommons.org/licenses/by/4.0/.

\section{References}

Audi, P. (2012). Grounding: Toward a theory of the in-virtue-of relation. The Journal of Philosophy, $109(12), 685-711$.

Baron, S. (2019). Counterfactual scheming. Mind, 129(514), 535-562.

Baron, S., Colyvan, M., \& Ripley, D. (2017). How mathematics can make a difference. Philosophers' Imprint, 17(3), 1-19.

Bennett, K. (2017). Making things up. Oxford and New York: Oxford University Press.

Bokulich, A. (2011). How scientific models can explain. Synthese, 180(1), 33-45.

Briggs, R. (2012). Interventionist couterfactuals. Philosophical Studies, 160(1), 139-166.

Cameron, R. (2010). On the source of necessity. In B. Hale \& A. Hoffmann (Eds.), Modality: Metaphysics, logic, and epistemology (pp. 137-151). Oxford and New York: Oxford University Press.

Correia, F., \& Schnieder, B. (2012). Grounding: An opinionated introduction. In B. Schnieder \& F. Correia (Eds.), Metaphysical grounding (pp. 1-36). Cambridge: Cambridge University Press.

Fine, K. (2010). Some puzzles of ground. Notre Dame Journal of Formal Logic, 51(1), 97-118.

Fine, K. (2012). A guide to ground. In F. Correia \& B. Schnieder (Eds.), Metaphysical grounding (pp. 37-80). Cambridge: Cambridge University Press.

Glazier, M. (2017). Essentialist explanation. Synthese, 174(11), 2871-2889.

Hempel, C. G. (1965). Studies in the logic of explanation. In C. G. Hempel (Ed.), Aspects of scientific explanation and other essays (pp. 245-296). New York: The Free Press.

Hitchcock, C. (1995). Salmon on explanatory relevance. Philosophy of Science, 62(2), 304-320.

Hitchcock, C. (2001). The intransitivity of causation revealed in equations and graphs. The Journal of Philosophy, 98(6), 273-299.

Jansson, L. (2018). When are structural equation models apt? Causation versus grounding. In A. Reutlinger \& J. Saatsi (Eds.), Explanation beyond causation: Philosophical perspectives on noncausal explanations (pp. 250-266). Oxford and New York: Oxford University Press.

Khalifa, K., Doble, G., \& Millson, J. (2018). Counterfactuals and explanatory pluralism. British Journal for the Philosophy of Science (forthcoming).

Kim, J. (1988). Explanatory realism, causal realism, and explanatory exclusion. Midwest Studies in Philosophy, 12(1), 225-239.

Kim, J. (1994). Explanatory knowledge and metaphysical dependence. Philosophical Issues, 5, 51-69.

Koslicki, K. (2016). Where grounding and causation part ways. Philosophical Studies, 173(1), 101-112.

Krämer, S., \& Roski, S. (2017). Difference-making grounds. Philosophical Studies, 174(5), 1191-1215.

Lewis, D. (1986). Causal explanation. In D. Lewis (Ed.), Philosophical Papers (Vol. II, pp. 214-240). Oxford and New York: Oxford University Press.

Lewis, D. (2000). Causation as influence. Journal of Philosophy, 97(4), 182-197.

Nickel, B. (2010). How general do theories of explanation have to be? Noûs, 44(6), 305-328.

Povich, M. (2018). Minimal models and the generalized ontic conception of scientific explanation. British Journal for the Philosophy of Science, 69(1), 117-137.

Povich, M. (2019). The narrow ontic counterfactual account of distinctively mathematical explanation. British Journal for the Philosophy of Science (forthcoming).

Raven, M. (2015). Ground. Philosophy Compass, 10(5), 322-333. 
Reutlinger, A. (2013). A theory of causation in the social and biological sciences. London: Palgrave Macmillan.

Reutlinger, A. (2016). Is there a monist theory of causal and non-causal explanation? Philosophy of Science, 83(5), 733-745.

Reutlinger, A. (2017). Does the counterfactual theory of explanation apply to non-causal explanations in metaphysics? European Journal for Philosophy of Science, 7(2), 239-256.

Reutlinger, A. (2018). Extending the couterfactual theory of explanation. In A. Reutlinger \& J. Saatsi (Eds.), Explanation beyond causation: Philosophical perspectives on non-causal explanations (pp. 74-95). Oxford and New York: Oxford University Press.

Rice, C. (2015). Moving beyond causes: Optimality models and scientific explanation. Noûs, 49(3), 589-615.

Rosen, G. (2010). Metaphysical dependence: Grounding and reduction. In B. Hale \& A. Hoffmann (Eds.), Modality: Metaphysics, logic, and epistemology (pp. 109-136). Oxford: Oxford University Press.

Rosen, G. (2017). Ground by law. Philosophical Issues, 27, 279-301.

Ruben, D.-H. (2012). Explaining explanation (updated and extended) (2nd ed.). Boulder: Paradigm Publishers.

Saatsi, J., \& Pexton, M. (2013). Reassessing woodward's account of explanation: Regularities, vounterfactuals, and noncausal explanations. Philosophy of Science, 80(5), 613-624.

Schaffer, J. (2016). Grounding in the image of causation. Philosophical Studies, 173(1), 49-100.

Schaffer, J. (2017). Laws for metaphysical explanation. Philosophical Issues, 27, 302-321.

Schnieder, B. (2011). A logic for 'because'. The Review of Symbolic Logic, 4, 445-465.

Tan, P. (2019). Counterpossible non-vacuity in scientific practice. Journal of Philosophy, 116(1), 32-60.

Weslake, B. (forthcoming). International studies in the philosophy of science.

Wilson, A. (2018a). Grounding entails counterpossible non-triviality. Philosophy and Phenomenological Research, 96(3), 716-728.

Wilson, A. (2018b). Metaphysical causation. Noûs, 52(4), 723-751.

Woodward, J. (1979). Scientific explanation. British Journal for the Philosophy of Science, 30(1), 41-67.

Woodward, J. (2003). Making things happen. Oxford and New York: Oxford University Press.

Publisher's Note Springer Nature remains neutral with regard to jurisdictional claims in published maps and institutional affiliations. 